\title{
DYNAMIC ANALYSIS OF BEAMS ON FRACTIONAL VISCOELASTIC FOUNDATION SUBJECT TO A VARIABLE SPEED MOVING LOAD
}

\author{
A. OUZIZI ${ }^{* *}$, F. ABDOUN ${ }^{1, * *}$, L. AZRAR ${ }^{1,2, * * *}$ \\ 1.Centre STIS, M2CS, ENSET, Mohammed V University, Rabat, Morocco \\ *anas.ouzizi@gmail.com; **farahabdoun@yahoo.fr \\ 2. Department of Mechanical Engineering, Faculty of Engineering, KAU, Jeddah, Saudi Arabia \\ ***1.azrar@um5s.net.ma
}

\begin{abstract}
The present paper investigates the dynamic response of beams resting on fractional viscoelastic foundation subjected to a moving load with variable speeds. The Galerkin with finite difference methods are used to deal with the governing equation of motion. The effect of various parameters, like fractional order derivative, foundation stiffness and damping, speed of moving load on the response of the beam are investigated and discussed.
\end{abstract}

\section{Keywords (12 bold): Moving loads; Variable} speed; Viscoelastic foundation; Vibration;

Fractional derivative.

\section{Introduction}

Recently, the dynamic response of beams on linear nonlinear viscoelastic foundations became one of important fields of research in the world. Specifically, when the Stephenson's bridge across river Dee Chester in England in 1947 collapsed, it encourages the engineers for research on moving load problems. Many problems of considerable practical importance can be related to the solution of beams on foundation. Reinforced concrete pavements of highways and airport runways and foundation slabs of buildings are well-known direct applications. Moving loads have a great effect on the bodies or structures over which it travels. Moreover, high-speed trains are becoming increasingly popular. For instance, the passage of the load with high speed will increase the deflection, due to this fact; we see that the study of moving loads is important for engineers.

Michaltsos [1], investigated dynamic response of beam on linear viscoelastic foundation subjected to moving load varying with time. Froio [2], analyzed the vibration of beams on nonlinear elastic foundations under harmonic moving load using finite element method and HHTimplementation (see Hilber et al. [3]) to deal with this problem. The fractional derivative was adopted in free vibration of beam by Ansari [4].
The paper focuses on the solution of a finite EulerBernoulli beam on a fractional viscoelastic foundation subjected to a variable speed moving load. Accent is on the fractional foundation and their effects.

The Galerkin method is used to solve the initial boundary value problem that governs the transverse vibration of the beam. Time response histories of the beam are graphically presented for various speeds of force and frequency. The effects of speed parameters and foundation stiffness are also investigated. As well as fractional derivative.

\section{Problem formulation}

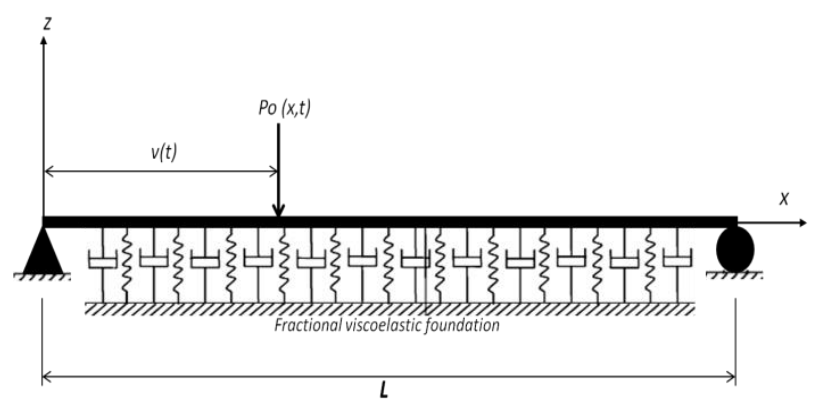

Fig. 1. Simply-supported beam on fractional viscoelastic foundation

Let us consider a beam of finite length $\mathrm{L}$ on a fractional viscoelastic foundation subjected to moving load with speed varying with time Fig 1 . The deformed of beam can be described by the transverse deflection $w(x, t)$ of the beam at point $x$ and time $t$. The dynamic behaviour is governed by the following partial differential equation:

$$
\begin{gathered}
E I \frac{\partial^{4} w(x, t)}{\partial x^{4}}+\rho A \frac{\partial^{2} w(x, t)}{\partial t^{2}}+P_{f}(x, t)=P_{0}(x, t) \\
=P_{0} \delta\left(x-x_{0}\right)
\end{gathered}
$$

Where $E, I, \rho$ and $A$ are the flexural rigidity, the density and the cross-sectional of the beam, respectively. $P(x, t)$ is a moving load with velocity $\mathrm{V}$ that is varying with time $t$ with the form $=V_{0} \pm a t$, where $V_{0}$ is initial velocity at $t=$ 
0 of the load when enters the beam and $a$ the constant acceleration or deceleration. Thus, the load moves at time $t$ a distance $x_{0}$ defined as: $x_{0}=V_{0} \pm\left(a t^{2} / 2\right)$.

$\delta$ denotes the Dirac delta function. $P_{f}(x, t)$ is the foundation taken as a linear Winkler foundation with linear stiffness and viscous damping that is generalized by the term containing fractional derivative as:

$P_{f}(x, t)=k_{L} w(x, t)+c_{f} \frac{\partial w(x, t)}{\partial t}+\mu D_{t}^{\alpha} w(x, t)$

In which $k_{L}, c_{f}$, are the linear and damping parameters of the foundation, respectively and $D_{t}^{\alpha}$ is the Caputo fractional derivative of order $\alpha \in(0,1)$ which is defined by the following convolution integrals [6].

$D_{t}^{\alpha}=\frac{d^{\alpha} f(t)}{d t^{\alpha}}=\frac{1}{\Gamma(1-\alpha)} \int_{0}^{t} \frac{d^{\prime}(u)}{(t-u)^{\alpha}} d u$

Where $\Gamma(z)$ is Gamma function that satisfies

$\Gamma(z)=\int_{0}^{\infty} t^{z-1} e^{-z} d t$

Boundary conditions and the initial conditions corresponding to the beam shown in Fig.1 are:

$\left.w(x, t)\right|_{0, L}=0,\left.\quad \frac{\partial^{2} w(x, t)}{\partial x^{2}}\right|_{0, L}=0$.

$w(x, t)||_{t=0}=0,\left.\quad \frac{\partial w(x, t)}{\partial x}\right|_{t=0}=0$.

\section{Solution of the problem}

For simply supported beams the deflection $w(x, t)$ is defined in a series form as:

$$
w(x, t)=\sum_{n=1}^{N} q_{n}(t) \sin \frac{n \pi x}{L}
$$

where $q_{n}(t)$ is the amplitude of the $n$th mode. Substituting Eqs. (2) and (6) into Eq. (1), multiplying both sides of Eq. (1) by $\sin (n \pi x / L)$ and integration over $[0, L]$.

The following differential equations are resulted

$$
\begin{aligned}
& \rho A \ddot{q}_{n}(t)+c_{f} \dot{q}_{n}(t)+\left[E I\left(\frac{n \pi}{L}\right)^{4}+k_{L}\right] q_{n}(t) \\
& +\mu D_{t}^{\alpha} q_{n}(t)=\frac{2 P_{0}}{L} \sin \left[\frac{n \pi}{L}\left(V_{0} \pm\left(\frac{a t^{2}}{2}\right)\right)\right]
\end{aligned}
$$

To deal with fractional derivative term of Eq. (7) we use a discrete approximation method for the computation of Caputo's fractional derivative given by the expression:

$D_{t}^{\alpha} q^{m}=\sigma_{\alpha, k} \sum_{j=1}^{m} \omega_{j}^{\alpha}\left(q^{m-j+1}-q^{m-j}\right)$

where

$\sigma_{\alpha, k}=\frac{1}{\Gamma(1-\alpha)} \frac{1}{(1-\alpha)} \frac{1}{k^{\alpha}}$ and $\omega_{j}^{\alpha}=j^{1-\alpha}-(j-1)^{1-\alpha}$

$k=\Delta t$ and $m$ is the number element of discrete approximation. The grid points in the time interval $[0, \mathrm{t}]$ are labelled $t_{m}=m \times k$. By using the expression (8) and considering and a second-order time central difference for the time derivative we get the recurrence equation:

$q_{m+1}=\left(\frac{2 \rho A}{\Delta t^{2}}+\mu \sigma_{\alpha, k} \omega_{1}^{\alpha}\right) q_{m}+\left(\frac{c_{f}}{2 \Delta t}-\frac{\rho A}{\Delta t^{2}}\right) q_{m-1}$
$-\mu D_{t}^{\alpha} q^{m}+\frac{2 P_{0}}{L} \sin \left[\frac{n \pi}{L}\left(V_{0} \pm\left(\frac{a t_{m+1}}{2}\right)^{2}\right)\right]$

This system is solved using an explicit time evaluation. In the numerical computation, the initial conditions are $q(0)=\dot{q}(0)=0$, the displacement of beam is obtained at any time $t$ and coordinate $x$. A MATLAB code is elaborated to compute and plot the deflection.

\section{Numerical results}

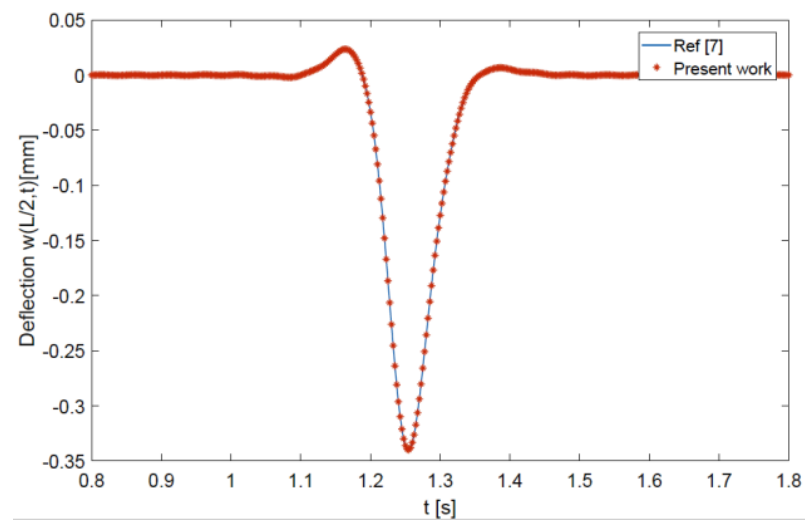

Fig. 2. Time response of simply supported beam at middle $\operatorname{span}\left(\mathrm{a}=0, V_{0}=20 \mathrm{~m} / \mathrm{s}\right)$.

The methodology presented in section 3 is verified here for the special case of non-fractional foundation when $(\alpha=1)$, $c_{f}=0$ and $\mu=c_{f}$, for which there are results in the literature for the case of moving load, Maximum deflection occurs at the mid-span of the beam, L/2. The results are presented at this point. Fig. 2 shows the comparison between results of Ref [7] and the present work for a linear foundation for time response of beam. The results show a perfect agreement with those in Ref [7], and this verifies the accuracy of presented method.

Another point is that for $\alpha=1$, the transverse displacement of the beam is close to be symmetrical but as the fractional order derivative used, the symmetry of the displacement is destroyed.

To discuss the accuracy and effects of different parameters of system, we will take a practical type of beams with data properties listed in Table. 1.

The effect of fractional order derivative on the vertical deflection is illustrated in Fig. 3. It can be seen that the fractional derivative order has a remarkable effect on the 
response. The transverse vibration amplitude decreases with increasing fractional order derivative.

Table. 1: Properties of UIC60 rail.

\begin{tabular}{lcc}
\hline \multicolumn{1}{c}{ Item } & Notation & Value \\
\hline Beam & & 50 \\
$\begin{array}{l}\text { Length } \\
\text { Young's modulus } \\
\text { (steel) }\end{array}$ & $L$ & $250 \mathrm{GPa}$ \\
$\begin{array}{l}\text { Mass density } \\
\text { Cross-sectional area }\end{array}$ & $\rho$ & $7850 \mathrm{~kg} / \mathrm{m}^{3}$ \\
$\begin{array}{l}\text { Second moment of } \\
\text { area }\end{array}$ & $I$ & $3.69 \times 10^{-3} \mathrm{~m}^{2}$ \\
Foundation & & \\
Linear stiffness & $k_{L}$ & $3.5 \times 10^{-5} \mathrm{~m}^{3}$ \\
Viscous damping & $c_{f}$ & $1732.5 \times 10^{3} \mathrm{Ns} / \mathrm{m}^{2}$ \\
Fractional coefficient & $\mu$ & $5 \times 10^{6} \mathrm{Ns}$ \\
Moving load & & $65 \mathrm{kN}$ \\
Load & $P_{0}$ & $20 \mathrm{~m} / \mathrm{s}$ \\
Initial velocity & $V_{0}$ & \\
\hline
\end{tabular}

Fig. 4, shows the deflection of the beam at mid-span with increasing value of acceleration and for different orders of $\alpha$. It can also seen from the Fig. 4 that increasing acceleration value of moving load results in early maximum deflection.

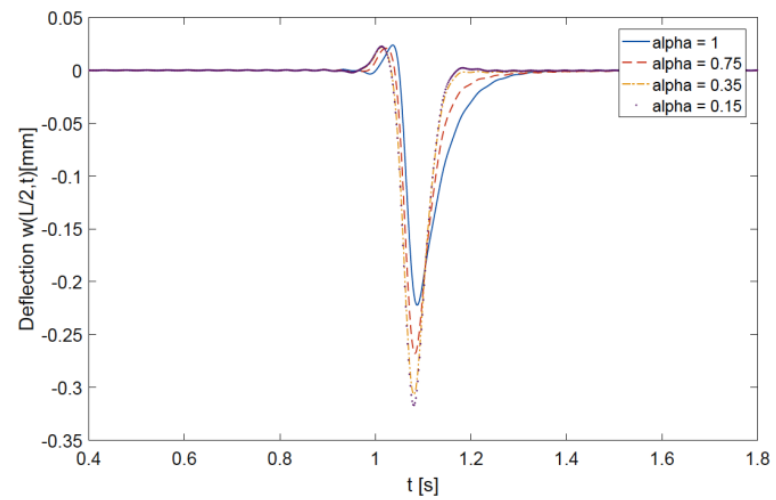

Fig. 3. Time response of simply supported beam at middle span for $a=6 \mathrm{~m} / \mathrm{s}$ and various value of order $\alpha=1 ; 0.75 ; 0.35$ and 0.15 .

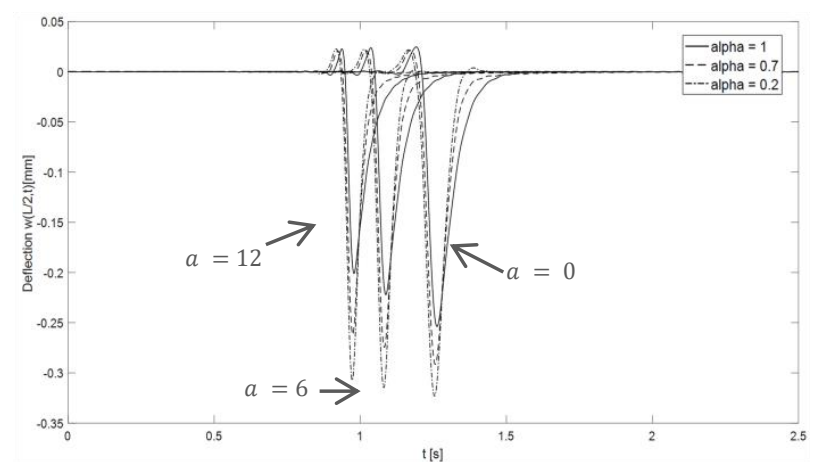

Fig. 4. Time response of simply supported beam at middle span for $V_{0}=20$ and various accelerations $a=0 ; 6$; and $12 \mathrm{~m} / \mathrm{s}$ and various value of order $\alpha=1 ; 0.7$; and 0.2 .

\section{Conclusion}

In this study, the dynamic response of beams on a Winkler fractional viscoelastic foundation subjected to moving load with variable speed is studied. A numerical code for analyzing the problem considering the fractional derivative has been elaborated.

The numerical results of the proposed method are verified for the case of non-fractional derivative and compared with results in the literatures and a good agreement was observed.

The effects of fractional derivative order on the vertical displacement of beam on Winkler viscoelastic foundation subjected to moving load are discussed.

\section{References}

[1] Michaltsos GT, Dynamic behavior of a singlespan beam subjected to loads moving with variable speeds, J Sound Vib 2002;258(2):359-72.

[2] D. Froio, Numerical dynamical analysis of beams on nonlinear elastic foundations under harmonic moving load, Proceedings, European Congress on Computational Methods in Applied Sciences and Engineering, Crete Island, Greece, 2016, Vol. 3, 4794-4809.

[3] H.M. Hilber, T.J.R. Hughes, R.L. Taylor, Improved numerical dissipation for time integration algorithms in structural dynamics, Earthquake Engineering and Structural Dynamics 1977;3(10), 283-292.

[4] R. Ansari, Free vibration of fractional viscoelastic Timoshenko nanobeams using the nonlocal elasticity theory, Physica E: Low-dimensional Systems and Nanostructures 2015; Vol.70, 318327.

[5] Ding $\mathrm{Hu}$, Chen Li-Qun, Yang Shao-Pu, Convergence of Galerkin truncation for dynamic response of finite beams on nonlinear foundations under a moving load, $\mathrm{J}$ Sound $\mathrm{Vib}$ 2012;331:2426e42.

[6] Diego A. Murio, Implicit finite difference approximation for time fractional diffusion equations, Computers and Mathematics with Applications 56 (2008) 1138-1145

[7] Senalp, A.D., Arikoglu, A., Ozkol, Dynamic response of a finite length Euler-Bernoulli beam on linear and nonlinear viscoelastic foundations to a concentrated moving force, J Mech Sci Technol (2010) 24: 1957. 